\title{
Lipschitz Estimates for Fractional Multilinear Singular Integral on Variable Exponent Lebesgue Spaces
}

\author{
Hui-Ling $\mathrm{Wu}^{1}$ and Jia-Cheng $\operatorname{Lan}^{2}$ \\ ${ }^{1}$ College of Education, Lishui University, Lishui 323000, China \\ ${ }^{2}$ College of Science, Lishui University, Lishui 323000, China \\ Correspondence should be addressed to Jia-Cheng Lan; jiachenglan@163.com
}

Received 6 February 2013; Accepted 11 August 2013

Academic Editor: Mustafa Bayram

Copyright (c) 2013 H.-L. Wu and J.-C. Lan. This is an open access article distributed under the Creative Commons Attribution License, which permits unrestricted use, distribution, and reproduction in any medium, provided the original work is properly cited.

We obtain the Lipschitz boundedness for a class of fractional multilinear operators with rough kernels on variable exponent Lebesgue spaces. Our results generalize the related conclusions on Lebesgue spaces with constant exponent.

\section{Introduction and Results}

Let $0<\alpha<n, \Omega \in L^{s}\left(S^{n-1}\right)(s>n /(n-\alpha))$ is homogeneous of degree zero on $R^{n}, S^{n-1}$ denotes the unit sphere in $R^{n}$, the fractional multilinear singular integral operator with rough kernel $T_{\Omega, \alpha, A}$ is defined by

$$
T_{\Omega, \alpha, A} f(x)=\int_{R^{n}} \frac{\Omega(x-y)}{|x-y|^{n-\alpha+m-1}} R_{m}(A ; x, y) f(y) d y,
$$

where $R_{m}(A ; x, y)$ denotes the $m$ th remainder of the Taylor series of a function $A$ defined on $R^{n}$ at $x$ about $y$. More precisely,

$$
R_{m}(A ; x, y)=A(x)-\sum_{|\gamma|<m} \frac{1}{\gamma !} D^{\gamma} A(y)(x-y)^{\gamma},
$$

and the corresponding fractional multilinear maximal operator is defined by

$$
\begin{aligned}
M_{\Omega, \alpha, A} f(x)= & \sup _{r>0} \frac{1}{r^{n-\alpha+m-1}} \\
& \times \int_{|x-y|<r}|\Omega(x-y)|\left|R_{m}(A ; x, y)\right||f(y)| d y .
\end{aligned}
$$

Multilinear operator was first introduced by Calderón in [1], and then Meyer [2] studied it in depth and extended such type of operators. Multilinear singular integral operator was later introduced by Professor Lu during 1999 [3]. Especially as $m=1$, the fractional multilinear singular integral operator $T_{\Omega, \alpha, A}$ is obviously the commutator operator

$$
\left[A, T_{\Omega, \alpha}\right] f(x)=A(x) T_{\Omega, \alpha} f(x)-T_{\Omega, \alpha}(A f)(x),
$$

the commutator is a typical non-convolution singular operator. Since the commutator has a close relation with partial differential equations and pseudo-differential operator, multilinear operator has been receiving more widely attention.

It is well known that the boundedness of $T_{\Omega, \alpha, A}$ and $M_{\Omega, \alpha, A}$ had been obtained on Lebesgue spaces in [4-7]. However, the corresponding results have not been obtained on $L^{p(\cdot)}\left(R^{n}\right)$. Nowadays, there is an evident increase of investigations related to both the theory of the spaces $L^{p(\cdot)}$ themselves and the operator theory in these spaces [8-11]. This is caused by possible applications to models with nonstandard local growth in elasticity theory, fluid mechanics, and differential equations [12-14]. The purpose of this paper is to study the behaviour of $T_{\Omega, \alpha, A}$ and $M_{\Omega, \alpha, A}$ on variable Lebesgue spaces.

To state the main results of this paper, we need to recall some notions. 
Definition 1. Suppose a measurable function $p(\cdot): R^{n} \rightarrow$ $[1, \infty)$, for some $\lambda>0$, then, the variable exponent Lebesgue space $L^{p(\cdot)}\left(R^{n}\right)$ is defined by

$$
L^{p(\cdot)}\left(R^{n}\right)=\left\{f \text { is measurable : } \int_{R^{n}}\left(\frac{|f(x)|}{\lambda}\right)^{p(x)} d x<\infty\right\},
$$

with norm

$$
\|f\|_{L^{p(\cdot)}\left(R^{n}\right)}=\inf \left\{\lambda>0: \int_{R^{n}}\left(\frac{|f(x)|}{\lambda}\right)^{p(x)} d x \leq 1\right\} .
$$

We denote

$$
\begin{aligned}
& p_{-}=\operatorname{essinf}\left\{p(x): x \in R^{n}\right\}, \\
& p_{+}=\operatorname{esssup}\left\{p(x): x \in R^{n}\right\} .
\end{aligned}
$$

Using this notation we define a class of variable exponent as follows:

$$
\Phi\left(R^{n}\right)=\left\{p(\cdot): R^{n} \longrightarrow[1, \infty), p_{-}>1, p_{+}<\infty\right\} .
$$

The exponent $p^{\prime}(\cdot)$ means the conjugate of $p(\cdot)$, namely, $1 / p(x)+1 / p^{\prime}(x)=1$ holds.

Definition 2. For $\beta>0$, the homogeneous Lipschitz space $\dot{\Lambda}_{\beta}$ is the space of functions $f$, such that

$$
\|f\|_{\dot{\Lambda}_{\beta}}=\sup _{x, h \in R^{n}, h \neq 0} \frac{\left|\Delta_{h}^{[\beta]+1} f(x)\right|}{|h|^{\beta}}<\infty
$$

where $\Delta_{h}^{1} f(x)=f(x+h)-f(x), \Delta_{h}^{k+1} f(x)=\Delta_{h}^{k} f(x+h)-$ $\Delta_{h}^{k} f(x), k \geq 1$.

Definition 3. For $0<\alpha<n$, the fractional integral operator with rough kernel is defined by

$$
\begin{gathered}
T_{\Omega, \alpha} f(x)=\int_{R^{n}} \frac{\Omega(x-y)}{|x-y|^{n-\alpha}} f(y) d y, \\
\bar{T}_{\Omega, \alpha} f(x)=\int_{R^{n}} \frac{|\Omega(x-y)|}{|x-y|^{n-\alpha}}|f(y)| d y .
\end{gathered}
$$

The corresponding fractional maximal operator with rough kernel is defined by

$$
M_{\Omega, \alpha} f(x)=\sup _{r>0} \frac{1}{r^{n-\alpha}} \int_{|x-y|<r}|\Omega(x-y)||f(y)| d y .
$$

When $\alpha=0, T_{\Omega, \alpha}$ is much more closely related to the elliptic partial equations of second order with variable coefficients. In 1955, Calderón and Zygmund [15] proved the $L^{p}$ boundedness. In 1971, Muckenhoupt and Wheeden [16] proved the $\left(L^{p}, L^{q}\right)$ boundedness of $T_{\Omega, \alpha}$ with power weights.

In this paper, we state some properties of variable exponents belonging to class $B\left(R^{n}\right)$.
Proposition 4. If $p(\cdot) \in \Phi\left(R^{n}\right)$ satisfies

$$
\begin{gathered}
|p(x)-p(y)| \leq \frac{-C}{\log (|x-y|)}, \quad|x-y| \leq \frac{1}{2}, \\
|p(x)-p(y)| \leq \frac{C}{\log (e+|x|)}, \quad|y| \geq|x|,
\end{gathered}
$$

Then, one has $p(\cdot) \in B\left(R^{n}\right)$.

Recently, Mitsuo Izuki has proved the condition as below.

Theorem A (see [17]). Suppose that $p(\cdot) \in \Phi\left(R^{n}\right)$ satisfies conditions (12) in Proposition 4. Let $0<\alpha<n / p_{+}$, and define the variable exponent $q(\cdot)$ by

$$
\frac{1}{p(x)}-\frac{1}{q(x)}=\frac{\alpha}{n}
$$

Then, one has that for all $f \in L^{p(\cdot)}\left(R^{n}\right)$,

$$
\left\|\left[b, I^{\alpha}\right] f\right\|_{L^{q()}\left(R^{n}\right)} \leq C\|b\|_{B M O\left(R^{n}\right)}\|f\|_{L^{p()}\left(R^{n}\right)}
$$

for all $f \in L^{p(\cdot)}\left(R^{n}\right)$ and $b \in B M O\left(R^{n}\right)$.

Next, we will discuss the boundedness of $T_{\Omega, \alpha, A}$ and $M_{\Omega, \alpha, A}$ on variable Lebesgue spaces. We can get $T_{\Omega, \alpha, A}$ and $M_{\Omega, \alpha, A}$ are bounded from $L^{p(\cdot)}\left(R^{n}\right)$ to $L^{q(\cdot)}\left(R^{n}\right)$. In fact, the results generalize Theorem A in [17] from classical Lebesgue spaces to variable exponent Lebesgue spaces. Now, let us formulate our results as follows.

Theorem 5. Suppose that $p(\cdot) \in \Phi\left(R^{n}\right)$ satisfies conditions (12) in Proposition 4. Let $0<\alpha<n / p_{+}, 0<\beta<1$, $0<\alpha+\beta<n / p_{+}$, and $1<p_{+}<n /(\alpha+\beta)$, and define the variable exponent $q(\cdot)$ by

$$
\frac{1}{q(x)}-\frac{1}{p(x)}=\frac{\alpha+\beta}{n} \text {. }
$$

If $D^{\gamma} A \in \dot{\Lambda}_{\beta}(|\gamma|=m-1)$, then, there is a $C>0$, independent of $f$ and $A$, such that

$$
\left\|T_{\Omega, \alpha, A} f\right\|_{L^{q(\cdot)}\left(R^{n}\right)} \leq C\left(\sum_{|\gamma|=m-1}\left\|D^{\gamma} A\right\|_{\dot{\Lambda}_{\beta}}\right)\|f\|_{L^{p(\cdot)}\left(R^{n}\right)} .
$$

Theorem 6. Suppose that $p(\cdot) \in \Phi\left(R^{n}\right)$ satisfies conditions (12) in Proposition 4. Let $0<\alpha<n / p_{+}, 0<\beta<1$, $0<\alpha+\beta<n / p_{+}$, and $1<p_{+}<n /(\alpha+\beta)$, and define the variable exponent $q(\cdot)$ by

$$
\frac{1}{q(x)}-\frac{1}{p(x)}=\frac{\alpha+\beta}{n} \text {. }
$$

If $D^{\gamma} A \in \dot{\Lambda}_{\beta}(|\gamma|=m-1)$, then, there is a $C>0$, independent of $f$ and $A$, such that

$$
\left\|M_{\Omega, \alpha, A} f\right\|_{L^{q(\cdot)}\left(R^{n}\right)} \leq C\left(\sum_{|\gamma|=m-1}\left\|D^{\gamma} A\right\|_{\Lambda_{\beta}}\right)\|f\|_{L^{p(\cdot)}\left(R^{n}\right)^{n}} .
$$

Remark 7. We point out that $C$ will denote positive constants whose values may change at different places. 


\section{Lemmas and Proof of Theorems}

Lemma 8 (see [15]). Let $A(x)$ be a function on $R^{n}$ with $m$ th order derivatives in $L_{l o c}^{l}\left(R^{n}\right)$ for somel $>n$. Then,

$$
\begin{aligned}
& \left|R_{m}(A ; x, y)\right| \\
& \quad \leq C|x-y|^{m} \sum_{|r|=m}\left(\frac{1}{\left|Q_{x}^{y}\right|} \int_{Q_{x}^{y}}\left|D^{\gamma} A(z)\right|^{l} d z\right)^{1 / l},
\end{aligned}
$$

where $Q_{x}^{y}$ is the cube centered at $x$ and having diameter $5 \sqrt{n}|x-y|$.

Lemma 9 (see [18]). For $0<\beta<1,1 \leq q<\infty$, we have

$$
\begin{aligned}
\|f\|_{\dot{\Lambda}_{\beta}} & =\sup _{\mathrm{Q}} \frac{1}{|Q|^{1+\beta / n}} \int_{Q}\left|f(x)-m_{Q}(f)\right| d x \\
& \approx \sup _{Q} \frac{1}{|Q|^{\beta / n}}\left(\frac{1}{|Q|} \int_{Q}\left|f(x)-m_{Q}(f)\right|^{q} d x\right)^{1 / q} .
\end{aligned}
$$

Lemma 10 (see [18]). Let $Q^{*} \subset Q, g \in \dot{\Lambda}_{\beta}(0<\beta<1)$, then,

$$
\left|m_{Q^{*}}(g)-m_{Q}(g)\right| \leq C|Q|^{\beta / n}\|g\|_{\dot{\Lambda}_{\beta}} .
$$

We state the following important lemma.

Lemma 11. Suppose $0<\alpha<n, 0<\beta<1$, with $0<\alpha+\beta<n$, $\Omega \in L^{s}\left(S^{n-1}\right)(s>n /(n-(\alpha+\beta))), D^{\gamma} A \in \dot{\Lambda}_{\beta}$. Then, there exists a constant $C$ only depends on $m, n, \alpha$, and $\beta$, such that

$$
\left|T_{\Omega, \alpha, A} f(x)\right| \leq C\left(\sum_{|\gamma|=m-1}\left\|D^{\gamma} A\right\|_{\Lambda_{\beta}}\right) \bar{T}_{\Omega, \alpha+\beta} f(x) .
$$

Proof. For any $x \in R^{n}$, let the cube be centered at $x$ and having the diameter be $l$, where $l>0$, we have

$$
\begin{array}{rl}
T_{\Omega, \alpha, A} & f(x) \\
& =\left(\int_{Q}+\int_{Q^{c}}\right) \frac{\Omega(x-y)}{|x-y|^{n-\alpha+m-1}} R_{m}(A ; x, y) f(y) d y \\
& :=H_{1}+H_{2} .
\end{array}
$$

Below, we give estimates of $H_{1}$. Let

$$
\begin{aligned}
\left|H_{1}\right| & \leq \sum_{j=0}^{\infty} \int_{2^{-j} Q \mid 2^{-j-1} Q} \frac{|\Omega(x-y)|\left|R_{m}(A ; x, y)\right|}{|x-y|^{n-\alpha+m-1}}|f(y)| d y \\
& \leq \sum_{j=0}^{\infty} \int_{2^{-j} Q \backslash 2^{-j-1} Q} \frac{|\Omega(x-y)|\left|R_{m}\left(A_{2^{-j} Q^{\prime}} ; x, y\right)\right|}{|x-y|^{n-\alpha+m-1}}|f(y)| d y .
\end{aligned}
$$

Note that $A_{2^{-j} Q}(y)=A(y)-\sum_{|\gamma|=m-1}(1 / \gamma !) m_{2^{-j} Q}\left(D^{\gamma} A\right) y^{r}$. When $y \in 2^{-j} Q \backslash 2^{-j-1} Q$, by Lemmas 8 , 9, and 10 , we have

$$
\left|R_{m}\left(A_{2^{-j} Q} ; x, y\right)\right| \leq C\left(2^{-j} l\right)^{\beta}|x-y|^{m-1} \sum_{|\gamma|=m-1}\left\|D^{\gamma} A\right\|_{\dot{\Lambda}_{\beta}} .
$$

Note that $|x-y| \geq 2^{-j-1}$, we have $|x-y|^{\beta} \geq 2^{-\beta}\left(2^{-j} l\right)^{\beta}$, such that

$$
\begin{aligned}
& \left|H_{1}\right| \leq C\left(\sum_{|\gamma|=m-1}\left\|D^{\gamma} A_{k}\right\|_{\dot{\Lambda}_{\beta}}\right) \\
& \times \sum_{j=0}^{\infty}\left(2^{-j} l\right)^{\beta} \int_{2^{-j} Q \mid 2^{-j-1} Q} \frac{|\Omega(x-y)||f(y)|}{|x-y|^{n-\alpha}} d y \\
& \leq C\left(\sum_{|\gamma|=m-1}\left\|D^{\gamma} A\right\|_{\dot{\Lambda}_{\beta}}\right) \\
& \times \sum_{j=0}^{\infty} \int_{2^{-j} Q \mid 2^{-j-1} Q} \frac{\left(2^{-j} l\right)^{\beta}|\Omega(x-y)||f(y)|}{|x-y|^{n-\alpha}} d y \\
& \leq C\left(\sum_{|\gamma|=m-1}\left\|D^{\gamma} A\right\|_{\Lambda_{\beta}}\right) \\
& \times \sum_{j=0}^{\infty} \int_{2^{-j} Q \mid 2^{-j-1} Q} \frac{2^{\beta}|x-y|^{\beta}|\Omega(x-y)|}{|x-y|^{n-\alpha}}|f(y)| d y \\
& \leq C\left(\sum_{|\gamma|=m-1}\left\|D^{\gamma} A\right\|_{\Lambda_{\beta}}\right) \int_{Q} \frac{|\Omega(x-y)||f(y)|}{|x-y|^{n-(\alpha+\beta)}} d y \\
& \leq C\left(\sum_{|\gamma|=m-1}\left\|D^{\gamma} A\right\|_{\dot{\Lambda}_{\beta}}\right) \int_{R^{n}} \frac{|\Omega(x-y)||f(y)|}{|x-y|^{n-(\alpha+\beta)}} d y \\
& =C\left(\sum_{|\gamma|=m-1}\left\|D^{\gamma} A\right\|_{\Lambda_{\beta}}\right) \bar{T}_{\Omega, \alpha+\beta} f(x) .
\end{aligned}
$$

Below, we give the estimates of $H_{2}$. For $0<\alpha+\beta<n$, we get

$$
\begin{aligned}
\left|H_{2}\right| & \leq \sum_{j=0}^{\infty} \int_{2^{j+1} Q \mid 2^{j} Q} \frac{|\Omega(x-y)|\left|R_{m}(A ; x, y)\right|}{|x-y|^{n-\alpha+m-1}}|f(y)| d y \\
& \leq \sum_{j=0}^{\infty} \int_{2^{j+1} Q \mid 2^{j} Q} \frac{|\Omega(x-y)|\left|R_{m}\left(A_{2^{j+1} \mathrm{Q}} ; x, y\right)\right|}{|x-y|^{n-\alpha+m-1}}|f(y)| d y .
\end{aligned}
$$

For any $y \in 2^{j+1} Q \backslash 2^{j} Q$,

$$
A_{2^{j+1} \mathrm{Q}}(y)=A(y)-\sum_{|\gamma|_{m-1}} \frac{1}{\gamma !} m_{2^{j+1} \mathrm{Q}}\left(D^{\gamma} A\right) .
$$

Thus, by Lemmas 8 and 9, we obtain

$$
\begin{aligned}
& \left|R_{m}\left(A_{2^{j+1} Q} ; x, y\right)\right| \\
& \quad \leq C\left(2^{j} l\right)^{\beta}|x-y|^{m-1} \sum_{|\gamma|=m-1}\left\|D^{\gamma} A\right\|_{\dot{\Lambda}_{\beta}} .
\end{aligned}
$$


And for $|x-y| \geq 2^{j} l$, we have $|x-y|^{\beta} \geq\left(2^{j} l\right)^{\beta}$. Hence,

$$
\begin{aligned}
& \left|H_{2}\right| \leq\left(\sum_{|\gamma|=m-1}\left\|D^{\gamma} A\right\|_{\dot{\Lambda}_{\beta}}\right) \\
& \times \sum_{j=0}^{\infty}\left(2^{j} l\right)^{\beta} \int_{2^{j+1} Q \backslash 2^{j} \mathrm{Q}} \frac{|\Omega(x-y)||f(y)|}{|x-y|^{n-\alpha}} d y \\
& \leq C\left(\sum_{|\gamma|=m-1}\left\|D^{\gamma} A\right\|_{\dot{\Lambda}_{\beta}}\right) \\
& \times \sum_{j=0}^{\infty} \int_{2^{j+1} \mathrm{Q} \backslash 2^{j} \mathrm{Q}} \frac{|x-y|^{\beta}|\Omega(x-y)||f(y)|}{|x-y|^{n-\alpha}} d y \\
& \leq C\left(\sum_{|\gamma|=m-1}\left\|D^{\gamma} A\right\|_{\dot{\Lambda}_{\beta}}\right) \\
& \times \sum_{j=0}^{\infty} \int_{2^{j+1} \mathrm{Q} \backslash 2^{j} \mathrm{Q}} \frac{|\Omega(x-y)||f(y)|}{|x-y|^{n-(\alpha+\beta)}} d y \\
& \leq C\left(\sum_{|\gamma|=m-1}\left\|D^{\gamma} A\right\|_{\dot{\Lambda}_{\beta}}\right) \int_{R^{n}} \frac{|\Omega(x-y)||f(y)|}{|x-y|^{n-(\alpha+\beta)}} d y \\
& \leq C\left(\sum_{|\gamma|=m-1}\left\|D^{\gamma} A\right\|_{\Lambda_{\beta}}\right) \bar{T}_{\Omega, \alpha+\beta} f(x) .
\end{aligned}
$$

From the proof above, we obtain

$$
\begin{aligned}
\left|T_{\Omega, \alpha, A} f(x)\right| & \leq\left|H_{1}\right|+\left|H_{2}\right| \\
& \leq C\left(\sum_{|\gamma|=m-1}\left\|D^{\gamma} A\right\|_{\dot{\Lambda}_{\beta}}\right) \bar{T}_{\Omega, \alpha+\beta} f(x) .
\end{aligned}
$$

Lemma 12 (see [19]). If $p(\cdot) \in \Phi\left(R^{n}\right)$, for all $f \in L^{p(\cdot)}\left(R^{n}\right)$, then, the norm $\|f\|_{L^{p(\cdot)}\left(R^{n}\right)}$ has the following equivalence:

$$
\begin{aligned}
\|f\|_{L^{p(\cdot)}\left(R^{n}\right)} & \leq \sup \left\{\int_{R^{n}}|f(x) g(x)| d x:\|g\|_{L^{p^{\prime}(\cdot)}\left(R^{n}\right)} \leq 1\right\} \\
& \leq r_{p}\|f\|_{L^{p^{\prime}(\cdot)}\left(R^{n}\right)^{\prime}}
\end{aligned}
$$

where $r_{p}:=1+1 / p_{-}-1 / p_{+}$.

Lemma 13 (see [19], the generalized Hölder inequality). If $p(\cdot) \in \Phi\left(R^{n}\right)$, then, for all $f \in L^{p(\cdot)}\left(R^{n}\right)$ and for all $g \in$ $L^{p^{\prime}(\cdot)}\left(R^{n}\right)$, we have

$$
\int_{R^{n}}|f(x) g(x)| d x \leq C\|f\|_{L^{p(\cdot)}\left(R^{n}\right)}\|g\|_{L^{p^{\prime}(\cdot)}\left(R^{n}\right)} .
$$

By a similar method of Ding and $\mathrm{Lu}[20]$, it is easy to verify the following result.
Lemma 14. For any $\varepsilon>0$ with $0<\alpha+\beta-\varepsilon<\alpha+\beta+\varepsilon<n$, we have

$$
\left|\bar{T}_{\Omega, \alpha+\beta} f(x)\right| \leq C\left[M_{\Omega, \alpha+\beta+\varepsilon} f(x)\right]^{1 / 2}\left[M_{\Omega, \alpha+\beta-\varepsilon} f(x)\right]^{1 / 2},
$$

where $C$ depends only on $\alpha, \beta, \varepsilon$, and $n$.

Lemma 15 (see [19]). Given that $p(\cdot): R^{n} \rightarrow[1, \infty)$, such that $p_{+}<\infty$, then, $\|f\|_{L^{p(\cdot)}\left(R^{n}\right)}<C_{1}$ if and only if $|f|_{L^{p(\cdot)}\left(R^{n}\right)}<$ $C_{2}$. In particular, if either constant equals 1 , one can make the other equals 1 as well.

Remark 16. We denote $|f|_{L^{p(\cdot)}\left(R^{n}\right)}=\int_{R^{n}}|f(y)|^{p(y)} d y$.

Lemma 17 (see [21]). Suppose that $p(\cdot) \in \Phi\left(R^{n}\right)$ satisfies conditions (12) in Proposition 4. Let $0<\alpha+\beta<n / p_{+}$, and define the variable exponent $q(\cdot)$ by

$$
\frac{1}{p(x)}-\frac{1}{q(x)}=\frac{\alpha+\beta}{n} \text {. }
$$

Then, one has that for all $f \in L^{p(\cdot)}\left(R^{n}\right)$,

$$
\left\|M_{\Omega, \alpha+\beta} f\right\|_{L^{q(\cdot)}\left(R^{n}\right)} \leq C\|f\|_{L^{p(\cdot)}\left(R^{n}\right)^{n}} .
$$

Lemma 18. Let $0<\alpha<n, \Omega \in L^{s}\left(S^{n-1}\right)$, then, for $x \in R^{n}$,

$$
\bar{T}_{\Omega, \alpha, A} f(x) \geq M_{\Omega, \alpha, A} f(x),
$$

where

$$
\bar{T}_{\Omega, \alpha, A} f(x)=\int_{R^{n}} \frac{|\Omega(x-y)|}{|x-y|^{n-\alpha+m-1}}\left|R_{m}(A ; x, y)\right||f(y)| d y .
$$

Proof. Since

$$
\begin{aligned}
& \bar{T}_{\Omega, \alpha, A} f(x) \\
& \quad=\int_{R^{n}} \frac{|\Omega(x-y)|}{|x-y|^{n-\alpha+m-1}}\left|R_{m}(A ; x, y)\right||f(y)| d y \\
& \quad \geq \int_{|x-y|<r} \frac{|\Omega(x-y)|}{|x-y|^{n-\alpha+m-1}}\left|R_{m}(A ; x, y)\right||f(y)| d y \\
& \quad \geq \frac{1}{r^{n-\alpha+m-1}} \int_{|x-y|<r}|\Omega(x-y)|\left|R_{m}(A ; x, y)\right||f(y)| d y,
\end{aligned}
$$

then,

$$
\bar{T}_{\Omega, \alpha, A} f(x) \geq M_{\Omega, \alpha, A} f(x) .
$$

Proof of Theorem 5. Since

$$
\left|T_{\Omega, \alpha, A} f(x)\right| \leq C\left(\sum_{|\gamma|=m-1}\left\|D^{\gamma} A\right\|_{\dot{\Lambda}_{\beta}}\right) \bar{T}_{\Omega, \alpha+\beta} f(x),
$$


by Lemma 12, then, we have

$$
\begin{aligned}
& \left\|T_{\Omega, \alpha, A} f(x)\right\|_{L^{q(\cdot)}\left(R^{n}\right)} \\
& \leq C\left(\sum_{|\gamma|=m-1}\left\|D^{\gamma} A\right\|_{\Lambda_{\beta}}\right) \\
& \quad \times \sup \left\{\int_{R^{n}} \bar{T}_{\Omega, \alpha+\beta} f(x)|g(x)| d x:\|g\|_{L^{q^{\prime}(\cdot)}\left(R^{n}\right)} \leq 1\right\} .
\end{aligned}
$$

Using the generalized Hölder inequality, then,

$$
\begin{aligned}
& \left\|T_{\Omega, \alpha, A} f\right\|_{L^{q(\cdot)}\left(R^{n}\right)} \\
& \quad \leq C\left(\sum_{|\gamma|=m-1}\left\|D^{\gamma} A\right\|_{\dot{\Lambda}_{\beta}}\right)\left\|\bar{T}_{\Omega, \alpha+\beta} f\right\|_{L^{q(\cdot)}\left(R^{n}\right)}\|g\|_{L^{q^{\prime}(\cdot)}\left(R^{n}\right)} \\
& \quad \leq C\left(\sum_{|\gamma|=m-1}\left\|D^{\gamma} A\right\|_{\dot{\Lambda}_{\beta}}\right)\left\|\bar{T}_{\Omega, \alpha+\beta} f\right\|_{L^{q(\cdot)}\left(R^{n}\right)} \cdot
\end{aligned}
$$

Next, we will prove $\left\|\bar{T}_{\Omega, \alpha+\beta} f\right\|_{L^{q(\cdot)}\left(R^{n}\right)} \leq\|f\|_{L^{p^{(\cdot)}\left(R^{n}\right)}}$. Fix $f \in L^{p(\cdot)}\left(R^{n}\right)$, without loss of generality we may assume that $\|f\|_{L^{p(\cdot)}\left(R^{n}\right)}=1$. Since $q_{+}<\infty$, by Lemma 15 it will suffice to prove that $\left|T_{\Omega, \alpha+\beta} f\right|_{L^{q(\cdot)}\left(R^{n}\right)} \leq C$.

Fix $\varepsilon, 0<\varepsilon<\min (\alpha+\beta, n-(\alpha+\beta))$, such that

$$
\frac{2}{\left(\varepsilon q_{+} / n\right)+1}>1 \text {, }
$$

define $r(\cdot): R^{n} \rightarrow[1,+\infty)$ by

$$
r(x)=\frac{2}{(\varepsilon q(x) / n)+1} .
$$

Then, by (44), we have $r_{-}>1$. Moreover, by elementary algebra, for all $x \in R^{n}$,

$$
\begin{aligned}
& \frac{1}{p(x)}-\frac{1}{r(x) q(x) / 2}=\frac{\alpha+\beta-\varepsilon}{n}, \\
& \frac{1}{p(x)}-\frac{1}{r^{\prime}(x) q(x) / 2}=\frac{\alpha+\beta+\varepsilon}{n} .
\end{aligned}
$$

So that by Lemma 14, we have

$$
\begin{aligned}
& \int_{R^{n}}\left|\bar{T}_{\Omega, \alpha+\beta} f(x)\right|^{q(x)} d x \\
& \quad \leq C \int_{R^{n}}\left[M_{\Omega, \alpha+\beta-\varepsilon} f(x)\right]^{q(x) / 2}\left[M_{\Omega, \alpha+\beta+\varepsilon} f(x)\right]^{q(x) / 2} d x .
\end{aligned}
$$

By Lemma 13, then,

$$
\begin{aligned}
\int_{R^{n}}\left|\bar{T}_{\Omega, \alpha+\beta} f(x)\right|^{q(x)} d x & \\
\leq & C\left\|\left[M_{\Omega, \alpha+\beta-\varepsilon} f(x)\right]^{q(x) / 2}\right\|_{L^{r(\cdot)}\left(R^{n}\right)} \\
& \times\left\|\left[M_{\Omega, \alpha+\beta+\varepsilon} f(x)\right]^{q(x) / 2}\right\|_{L^{r^{\prime}(\cdot)}\left(R^{n}\right)} .
\end{aligned}
$$

Without loss of generality, we may assume that each is greater than 1 , since, otherwise, there is nothing to prove. In this case, in the definition of each norm we may assume that the infimum is taken over by values of $\lambda$ which are greater than 1. But then, since for all $x \in R^{n}$ and $\lambda>1, \lambda^{2 / q(x)} \geq \lambda^{2 / q_{+}}$, we have

$$
\begin{aligned}
& \int_{R^{n}}(\left.\frac{\left[M_{\Omega, \alpha+\beta-\varepsilon} f(x)\right]^{q(x) / 2}}{\lambda}\right)^{r(x)} d x \\
& \quad=\int_{R^{n}}\left(\frac{M_{\Omega, \alpha+\beta-\varepsilon} f(x)}{\lambda^{2 / q(x)}}\right)^{r(x) q(x) / 2} d x \\
& \quad \leq \int_{R^{n}}\left(\frac{M_{\Omega, \alpha+\beta-\varepsilon} f(x)}{\lambda^{2 / q_{+}}(x)}\right)^{r(x) q(x) / 2} d x .
\end{aligned}
$$

Therefore, by (46) and Lemma 17, we have

$$
\begin{aligned}
\left\|\left[M_{\Omega, \alpha+\beta-\varepsilon} f(x)\right]^{q(x) / 2}\right\|_{L^{r(x)}\left(R^{n}\right)} & \leq\left\|\left[M_{\Omega, \alpha+\beta-\varepsilon} f(x)\right]\right\|_{L^{r(x) q(x) / 2}\left(R^{n}\right)}^{q_{+} / 2} \\
& \leq C\|f\|_{L^{p(x)}\left(R^{n}\right)}^{q_{+} / 2} \leq C .
\end{aligned}
$$

In the same way, we have

$$
\begin{aligned}
& \int_{R^{n}}\left(\frac{\left[M_{\Omega, \alpha+\beta+\varepsilon} f(x)\right]^{q(x) / 2}}{\lambda}\right)^{r^{\prime}(x)} d x \\
& \quad=\int_{R^{n}}\left(\frac{M_{\Omega, \alpha+\beta+\varepsilon} f(x)}{\lambda^{2 / q(x)}}\right)^{r^{\prime}(x) q(x) / 2} d x \\
& \quad \leq \int_{R^{n}}\left(\frac{M_{\Omega, \alpha+\beta+\varepsilon} f(x)}{\lambda^{2 /(q)_{+}(x)}}\right)^{r^{\prime}(x) q(x) / 2} d x .
\end{aligned}
$$

Therefore, by (47) and Lemma 17, then,

$$
\begin{aligned}
\left\|\left[M_{\Omega, \alpha+\beta+\varepsilon} f(x)\right]^{q(x) / 2}\right\|_{L^{r^{\prime}(x)}\left(R^{n}\right)} & \leq\left\|\left[M_{\Omega, \alpha+\beta+\varepsilon} f(x)\right]\right\|_{L^{r^{\prime}(x) q(x) / 2}\left(R^{n}\right)}^{q_{+} / 2} \\
& \leq C\|f\|_{L^{p(x)}\left(R^{n}\right)}^{q_{+} / 2} \leq C .
\end{aligned}
$$

Hence,

$$
\left|\bar{T}_{\Omega, \alpha+\beta} f\right|_{L^{q(\cdot)}\left(R^{n}\right)}=\int_{R^{n}}\left|\bar{T}_{\Omega, \alpha+\beta} f(x)\right|^{q(x)} d x \leq C .
$$

So, we have

$$
\begin{gathered}
\left\|\bar{T}_{\Omega, \alpha+\beta} f\right\|_{L^{q(\cdot)}\left(R^{n}\right)} \leq\|f\|_{L^{p(\cdot)}\left(R^{n}\right)}, \\
\left\|T_{\Omega, \alpha, A} f\right\|_{L^{q(\cdot)}\left(R^{n}\right)} \\
\leq C\left(\sum_{|\gamma|=m-1}\left\|D^{\gamma} A\right\|_{\Lambda_{\beta}}\right)\left\|\bar{T}_{\Omega, \alpha+\beta} f\right\|_{L^{q(\cdot)}\left(R^{n}\right)} \\
\leq C\left(\sum_{|\gamma|=m-1}\left\|D^{\gamma} A\right\|_{\Lambda_{\beta}}\right)\|f\|_{L^{p(\cdot)}\left(R^{n}\right)^{\prime}}
\end{gathered}
$$

This completes the proof of Theorem 5 . 
By Lemmas 15 and 18 and Theorem 5, the proof of Theorem 6 is directly deduced.

\section{Acknowledgments}

This paper is supported by the Natural Science Foundation of Zhejiang Province (M6090681) and supported by the Education Deptartment of Zhejiang Province (Y201120509).

\section{References}

[1] A. P. Calderón, "Algebras of singular integral operators," in Proceedings of Symposia in Pure Mathematics, vol. 10, pp. 18-55, American Mathematical Society, Providence, RI, USA, 1967.

[2] Y. Meyer, Ondelettes et Opérateurs. I, Hermann, Paris, France, 1990.

[3] S. Lu, "Multilinear oscillatory integrals with CalderónZygmund kernel," Science in China A, vol. 42, no. 10, pp. 1039-1046, 1999.

[4] J. C. Lan, "Uniform boundedness of multilinear fractional integral operators," Applied Mathematics, vol. 21, no. 3, pp. 365$372,2006$.

[5] S. Lu and P. Zhang, "Lipschitz estimates for generalized commutators of fractional integrals with rough kernel," Mathematische Nachrichten, vol. 252, pp. 70-85, 2003.

[6] Y. Ding, "A note on multilinear fractional integrals with rough kernel," Advances in Mathematics, vol. 30, no. 3, pp. 238-246, 2001.

[7] X. X. Tao and Y. P. Wu, "BMO estimates for multilinear fractional integrals," Analysis in Theory and Applications, vol. 28, pp. 224-231, 2012.

[8] V. Kokilashvili and S. Samko, "On sobolev theorem for RieszType potentials in Lebesgue spaces with variable exponent," Journal for Analysis and its Applications, vol. 22, no. 4, pp. 899910, 2003.

[9] L. Diening, "Maximal function on Musielak-Orlicz spaces and generalized Lebesgue spaces," Bulletin des Sciences Mathematiques, vol. 129, no. 8, pp. 657-700, 2005.

[10] D. Cruz-Uribe, A. Fiorenza, J. M. Martell, and C. Pérez, "The boundedness of classical operators on variable $L^{p}$ spaces," Annales Academiae Scientiarum Fennicae Mathematica, vol. 31, no. 1, pp. 239-264, 2006.

[11] W. Wang and J. Xu, "Commutators of multilinear singular integrals with Lipschitz functions on products of variable exponent Lebesgue spaces," Advances in Mathematics, vol. 38, no. 6, pp. 669-677, 2009.

[12] E. Acerbi and G. Mingione, "Regularity results for stationary electro-rheological fluids," Archive for Rational Mechanics and Analysis, vol. 164, no. 3, pp. 213-259, 2002.

[13] M. Ružička, Electroreological Fluids: Modeling and Mathematical Theory, vol. 1748 of Lecture Notes in Mathematics, 2000.

[14] E. Acerbi and G. Mingione, "Regularity results for a class of functionals with non-standard growth," Archive for Rational Mechanics and Analysis, vol. 156, no. 2, pp. 121-140, 2001.

[15] A. P. Calderón and A. Zygmund, "On a problem of Mihlin," Transactions of the American Mathematical Society, vol. 78, pp. 209-224, 1955.

[16] B. Muckenhoupt and R. L. Wheeden, "Weighted norm inequalities for singular and fractional integrals," Transactions of the American Mathematical Society, vol. 161, pp. 249-258, 1971.
[17] M. Izuki, "Commutators of fractional integrals on Lebesgue and Herz spaces with variable exponent," Rendiconti del Circolo Matematico di Palermo, vol. 59, no. 3, pp. 461-472, 2010.

[18] A. P. Calderón and A. Zygmund, "On singular integral with variable kernels," Journal of Applied Analysis, vol. 7, pp. 221-238, 1978.

[19] O. Kováčik and J. Rákosník, "On spaces $L^{p(x)}$ and $W^{k, p(x), "}$ Czechoslovak Mathematical Journal, vol. 41, no. 4, pp. 592-618, 1991.

[20] Y. Ding and S. Lu, "Higher order commutators for a class of rough operators," Arkiv for Matematik, vol. 37, no. 1, pp. 33-44, 1999.

[21] H. L. Wu and J. C. Lan, "The boundedness of rough fractional integral operators on variable exponent Lebesgue spaces," Anyalsis in Theory and Applications, vol. 28, pp. 286-293, 2012. 


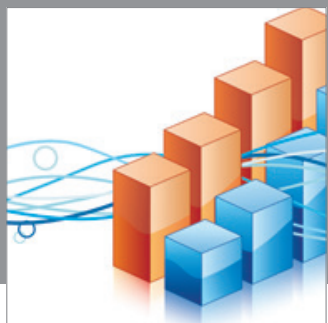

Advances in

Operations Research

mansans

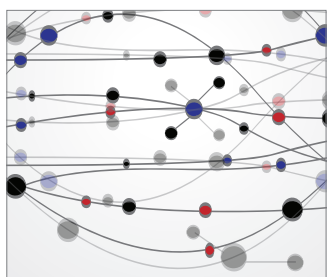

The Scientific World Journal
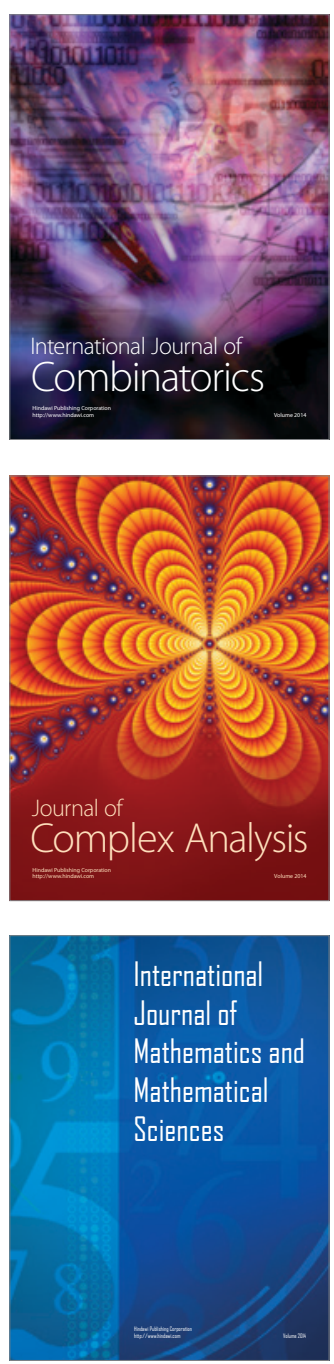
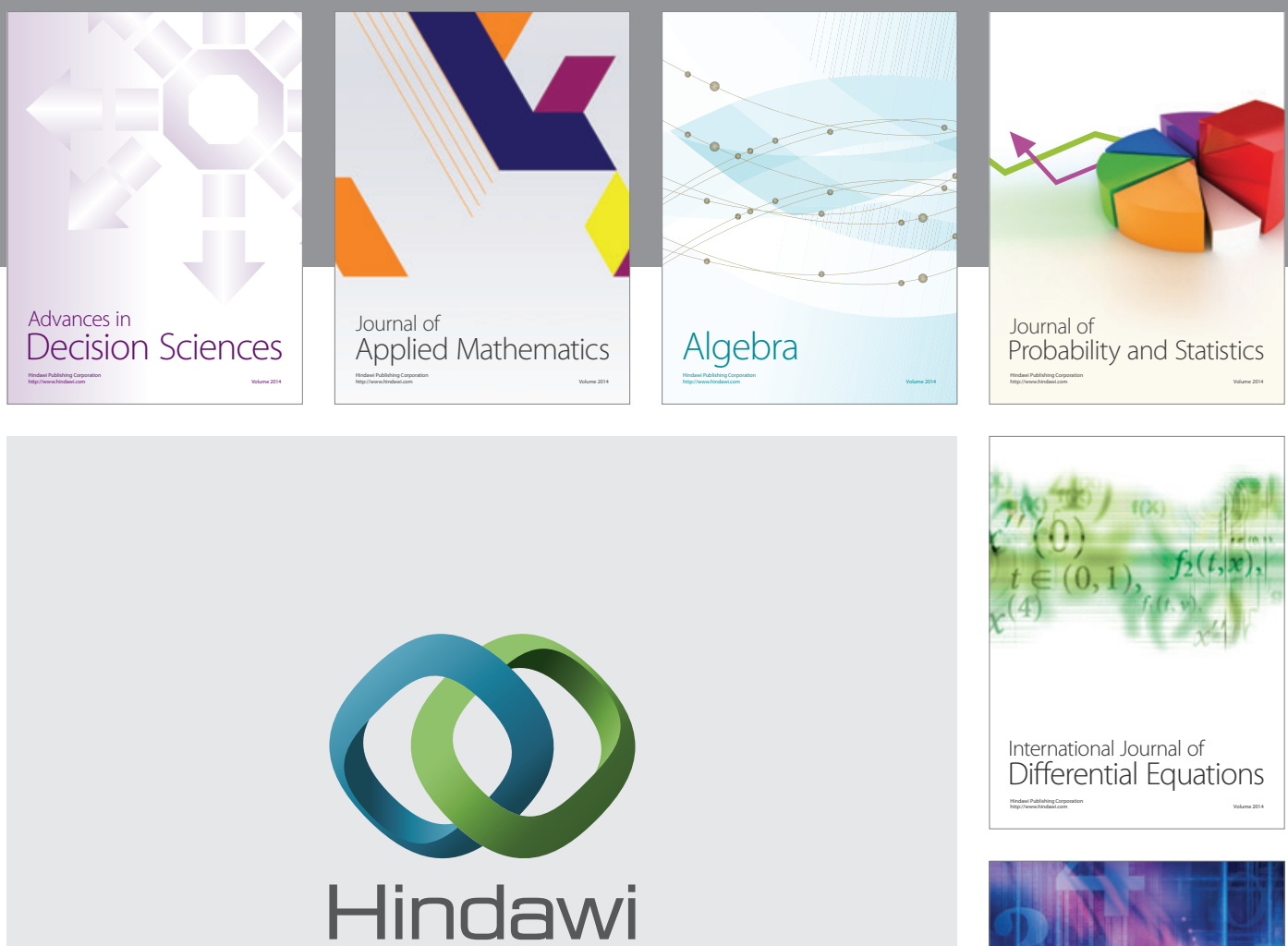

Submit your manuscripts at http://www.hindawi.com
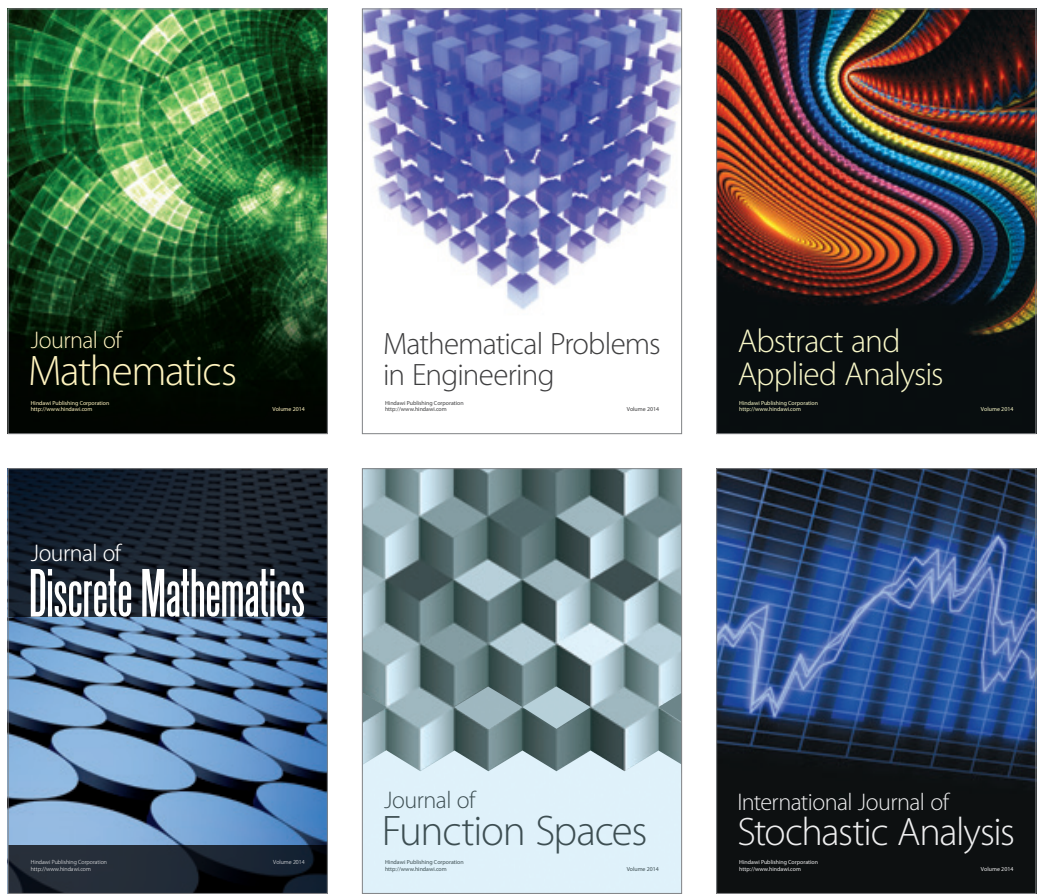

Journal of

Function Spaces

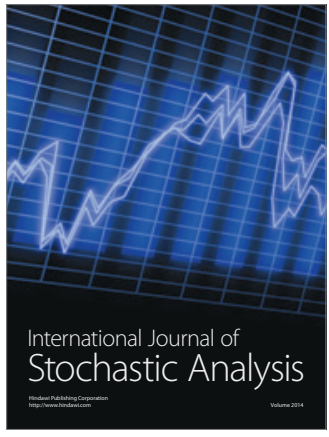

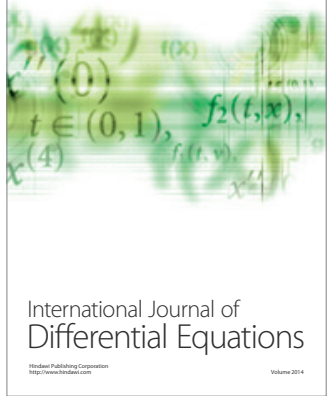
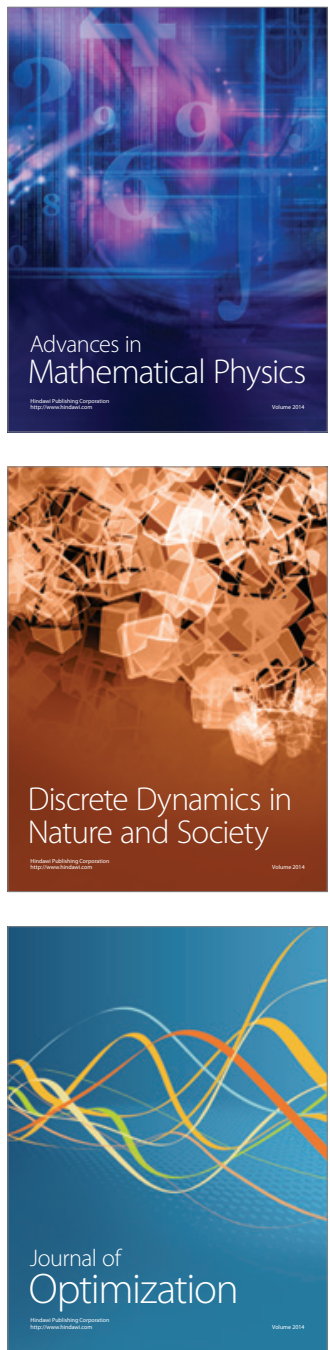